\title{
Beta-Blocker Therapy in Severe Traumatic Brain Injury: A Prospective Randomized Controlled Trial
}

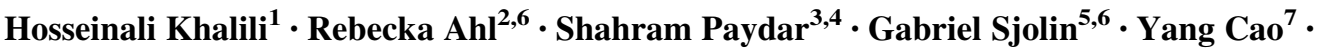 \\ Hossein Abdolrahimzadeh Fard $^{2,3} \cdot$ Amin Niakan $^{1} \cdot$ Kamil Hanna $^{8} \cdot$ Bellal Joseph $^{8}$. \\ Shahin Mohseni ${ }^{6,9}$
}

Published online: 30 January 2020

(C) The Author(s) 2020

\begin{abstract}
Background Observational studies have demonstrated improved outcomes in TBI patients receiving in-hospital betablockers. The aim of this study is to conduct a randomized controlled trial examining the effect of beta-blockers on outcomes in TBI patients.

Methods Adult patients with severe TBI (intracranial AIS $\geq 3$ ) were included in the study. Hemodynamically stable patients at $24 \mathrm{~h}$ after injury were randomized to receive either $20 \mathrm{mg}$ propranolol orally every $12 \mathrm{~h}$ up to 10 days or until discharge $(\mathrm{BB}+)$ or no propranolol $(\mathrm{BB}-)$. Outcomes of interest were in-hospital mortality and Glasgow Outcome Scale-Extended (GOS-E) score on discharge and at 6-month follow-up. Subgroup analysis including only isolated severe TBI (intracranial AIS $\geq 3$ with extracranial AIS $\leq 2$ ) was carried out. Poisson regression models were used.

Results Two hundred nineteen randomized patients of whom $45 \%$ received $\mathrm{BB}$ were analyzed. There were no significant demographic or clinical differences between $\mathrm{BB}^{+}$and $\mathrm{BB}^{-}$cohorts. No significant difference in inhospital mortality (adj. IRR 0.6 [95\% CI 0.3-1.4], $p=0.2$ ) or long-term functional outcome was measured between the cohorts $(p=0.3)$. One hundred fifty-four patients suffered isolated severe TBI of whom $44 \%$ received BB. The $\mathrm{BB}^{+}$group had significantly lower mortality relative to the $\mathrm{BB}^{-}$group $(18.6 \%$ vs. $4.4 \%, \mathrm{p}=0.012)$. On regression analysis, propranolol had a significant protective effect on in-hospital mortality (adj. IRR $0.32, p=0.04$ ) and functional outcome at 6 -month follow-up (GOS-E $\geq 5$ adj. IRR 1.2, $p=0.02$ ).

Conclusion Propranolol decreases in-hospital mortality and improves long-term functional outcome in isolated severe TBI. This randomized trial speaks in favor of routine administration of beta-blocker therapy as part of a standardized neurointensive care protocol.

Level of evidence Level II; therapeutic.

Study type Therapeutic study.
\end{abstract}

This manuscript was presented as a Podium Presentation at the American Association for the Surgery of Trauma (AAST) annual meeting 2019 in Dallas, USA.

Shahin Mohseni

mohsenishahin@yahoo.com; shahin.mohseni@oru.se

Hosseinali Khalili

khalilih@yahoo.com

Rebecka Ahl

rebecka.ahl@cantab.net
Shahram Paydar

paydarsh@gmail.com

Gabriel Sjolin

gabriel.sjolin@regionorebrolan.se

Yang Cao

yang.cao@oru.se 


\section{Introduction}

Traumatic injury is one of the most common causes of death in people under the age of forty worldwide, and onethird of all trauma-related deaths are a result of intracranial insults [1]. For survivors of severe brain injury, permanent neurologic consequences often ensue. Intracranial injury is the product of a primary hit and a secondary hit. The primary injury occurs at the time of trauma and can only be addressed through preventative measures. Secondary injury is caused by complications of the primary insult and is driven through processes such as hypoxia, cerebral edema and ischemia $[2,3]$. Research is consequently focused on measures that can reduce the incidence of secondary injury processes to improve survival and functional outcome after traumatic brain injury (TBI).

International guidelines have been formed to standardize intensive care management for brain trauma victims [4, 5]. This includes standardized neurointensive care, the use of intracranial pressure monitoring, neurospecific monitoring and neurosurgical intervention [4]. The utility of early beta-blocker therapy in TBI has been demonstrated in several retrospective as well as prospective observational studies demonstrating beneficial effects on clinical outcomes and survival [6-10]. It is hypothesized that the adrenergic storm induced by the initial insult may worsen the secondary brain injury through mechanisms of cerebral vasoconstriction and subsequent ischemia [11, 12]. Till this date, no randomized controlled trial assessing the impact of beta-blockade on mortality in the context of TBI has been performed. However, significant improvements in neuroprotective care protocols have been introduced since the 1980s. The aim of this study is to conduct a randomized controlled trial examining the effect of beta-blockers on outcomes in TBI patients. We hypothesized that betablockers improve survival and functional outcomes.

Hossein Abdolrahimzadeh Fard

Dr.h.a.fard@gmail.com

Amin Niakan

aminniakan@yahoo.com

Kamil Hanna

kamilhanna@surgery.arizona.edu

Bellal Joseph

bjoseph@surgery.arizona.edu

1 Department of Neurosurgery, Trauma Research Center, Shahid Rajaee (Emtiaz) Trauma Hospital, Shiraz University of Medical Sciences, Shiraz, Iran

2 Department of Surgery, Karolinska University Hospital, Stockholm, Sweden

3 Trauma Research Center, Rajaee (Emtiaz) Trauma Hospital, Shiraz University of Medical Sciences, Shiraz, Iran
Methods

\section{Patient inclusion and exclusion criteria}

The trial ("Effect of Propranolol in Traumatic Brain Injury") was registered at Iranian Registry of Clinical Trials (IRCTID: 20130310012776N4) and was approved by the institutional review board (IRB number IR.SUMS.REC.1396-133). This study is a single-center non-blinded randomized trial comparing the effectiveness of propranolol on overall outcomes in patients who suffered a severe TBI (intracranial Abbreviated Injury Scale (AIS) score $\geq 3$ ) in addition to standard neurointensive care. All adult patients (age $\geq 18$ years) admitted to the neurosurgical intensive care unit (NICU) of Shaheed Rajee (Emtiaz) trauma hospital, Shiraz University Hospital, Shiraz, Iran, between December 1, 2017 and August 31, 2018, were prospectively screened for inclusion in the current study. The main NICU admission criteria at our institution are a severe TBI detected on admission CT or GCS score of $<12$ for observation and monitoring or neurosurgical interventions. Patients who suffered a blunt traumatic brain injury without any extracranial injury requiring a surgical intervention (e.g., thoracotomy/sternotomy, emergency laparotomy, pelvic packing, surgery for spinal cord injury and long-bone fractures) within $24 \mathrm{~h}$ of admission were included in the study. Patients on pre-injury beta-blocker therapy, persistent shock (systemic blood pressure $<100 \mathrm{mmHg}$, base deficit $>4$, or oliguria) at $24 \mathrm{~h}$ after admission, and those transferred from another hospital were not eligible for inclusion.

\section{Data collection}

Data collection included the following covariates: age, sex, co-morbidities (hypertension, diabetes mellitus, cardiovascular

4 Department of Surgery, Shiraz University of Medical Sciences, Shiraz, Iran

5 Department of Surgery, Orebro University Hospital, 70185 Örebro, Sweden

6 School of Medical Sciences, Orebro University, 70281 Örebro, Sweden

7 Clinical Epidemiology and Biostatistics, School of Medical Sciences, Orebro University, 70182 Örebro, Sweden

8 Department of Surgery, University of Arizona College of Medicine, Tucson, AZ, USA

9 Division of Trauma and Emergency Surgery, Department of Surgery, Orebro University Hospital, 70185 Örebro, Sweden 
disease [CVD]), admission systolic blood pressure (SBP), Glasgow Coma Scale (GCS) score on admission, AIS for all body regions, Injury Severity Score (ISS), type of intracranial injury, neurosurgical intervention, extended Glasgow Outcome Scale (GOS-E) score at discharge and at 6 months post-discharge. Patients included in the study were followed throughout their hospital stay and evaluated at a follow-up visit in the clinic after 6 months.

\section{Beta-blocker administration protocol}

Patients with a severe intracranial injury were randomized to either beta-blocker $\left(\mathrm{BB}^{+}\right)$or no beta-blocker $\left(\mathrm{BB}^{-}\right)$ therapy by random number generator $24 \mathrm{~h}$ after admission if hemodynamically stable (defined as systolic blood pressure over $100 \mathrm{mmHg}$ ), not requiring any vasopressor support or blood transfusion and if allowed to start enteral feeding. Severe traumatic brain injury was defined as an intracranial injury yielding an Abbreviated Injury Scale score of 3-5 on initial brain computer tomography (CT) evaluated by an attending radiologist and neurosurgeon within $24 \mathrm{~h}$ of admission. The decision to only include severe TBI was based on the fact that the catecholamine surge after brain injury has been correlated with the severity of the intracranial injury and neurologic deficit [13]. Furthermore, a correlation between the excessive serum catecholamines proportional to the severity of the intracranial injury influencing overall clinical outcome has previously been noted [14].

Patients who were randomized to the beta-blocker therapy group received $20 \mathrm{mg}$ of oral propranolol. In cases where patients could not obey command or were intubated, the medication was administered through a nasogastric tube. This dose was repeated every $12 \mathrm{~h}$ up to 10 days following injury or until hospital discharge. All patients were observed in NICU at least $24 \mathrm{~h}$ after initiation of betablocker therapy with continuous blood pressure and heart rate monitoring. Patients who received beta-blocker and developed bradycardia (heart rate $<50 \mathrm{bpm}$ ), hypotension (systolic blood pressure $<100 \mathrm{~mm} \mathrm{Hg}$ ) or refused to continue treatment during the 10-day intervention period were excluded from further analysis. Propranolol was used as the preferred beta-blocker therapy due to its longstanding use in patients suffering subarachnoid hemorrhage and stroke where a decreased oxygen consumption, carbon dioxide production and glucose consumption have been measured [15-18]. Additionally, propranolol's hydrophilic profile allows it to cross the blood-brain barrier more readily than some other beta-blockers.

Propranolol has also previously been shown to decrease the risk for adverse outcomes and safe to use early after the insult in TBI patients $[8,9,18]$. It has been suggested that the hyperadrenergic state is most pronounced during the first week after traumatic insult [14, 19]. Hence, the decision was made to continue the beta-blocker treatment for at least 10 days in patients who were still admitted to hospital due to their TBI.

\section{Ethical considerations}

The ethics committee did consent to the use of blinding and placebo for this trial; however, due to insufficient funding, the decision was made to continue with the study without blinding and the use of placebo. Consent for inclusion was sought from patients themselves or, if required, their next of kin. The study adheres to the CONSORT statement.

\section{Outcomes}

The primary outcome of interest was in-hospital mortality. The secondary outcomes were GOS-E on discharge and at 6-month follow-up.

\section{Power analysis}

We calculated that 210 randomized patients would provide $80 \%$ power at a significance level of $5 \%$ to detect at $65 \%$ relative risk reduction, assuming an event rate of $20 \%$ in the control group.

\section{Statistical analysis}

For analysis purposes, several continuous variables were dichotomized using clinically relevant cutoff points (age $\geq 55$ vs. $\leq 54$ years; GCS score $\geq 9$ vs. $\leq 8$; head AIS 3 vs. $\geq 4$; and ISS $<15$ vs. $\geq 16$ ). Good functional outcome was set to an GOS-E $\geq 5$. Demographics and clinical characteristics were compared between patients who received beta-blocker therapy versus those who did not. Chi-square or two-sided Fisher's exact test was used for comparison of categorical variables, while Student's t test or Mann-Whitney $U$ test were used for comparison of continuous variables when appropriate. Values are reported as mean with standard deviation (SD) for continuous variables and as percentages for categorical variables.

Association between beta-blocker therapy and in-hospital survival, GOS-E at discharge and at 6 months were evaluated using Poisson regression models with robust standard errors. Potential confounding was adjusted for by including the following covariates in the model: age, sex, hypertension, diabetes mellitus, CVD, GCS, head AIS, ISS, type of intracranial injury/injuries and neurosurgical intervention. Results are reported as adjusted incidence rate ratios (IRRs) with corresponding 95\% confidence intervals (CI). A two-sided $p$ value of less than 0.05 was considered statistically significant. All statistical analyses were 
performed using the SPSS Windows version 25.0 (SPSS Inc., Chicago, IL).

Patients with isolated severe brain injury, defined by an intracranial injury yielding a head AIS score of $\geq 3$ with an AIS $\leq 2$ in all other body regions, were put forward for further analysis to better reflect the effect of propranolol on the outcomes of interest in patients with isolated severe TBI.

\section{Results}

During the 8-month study period, a total of 356 patients were admitted to our NICU of whom 240 patients met inclusion criteria. Eighteen of them did not consent to inclusion in the study leaving a total of 222 patients eligible for randomization of whom 120 patients were allocated to the $\mathrm{BB}^{-}$arm and 102 patients to the $\mathrm{BB}^{+}$arm.
Three patients were excluded from the $\mathrm{BB}^{+}$group due to bradycardia (heart rate $<50 \mathrm{bpm}$ ) leaving 99 patients in the $\mathrm{BB}^{+}$group (see Fig. 1 for CONSORT diagram) [20]. Three patients in this group were lost to follow-up. As depicted in Table 1, there was no statistical difference in patient demographics and clinical characteristics between the cohorts. Death occurred in $16.7 \%(n=20)$ of patients allocated to the $\mathrm{BB}^{-}$group with a corresponding percentage of $8.1 \%(n=8)$ in the $\mathrm{BB}^{+}$group $(p=0.058)$. No difference in good functional outcome (GOS-E $\geq 5$ ) at discharge $(p=0.36)$ or at 6-month follow-up $(p=0.09)$ could be detected between the cohorts (Table 1). After adjusting for all clinical variables between the groups, no difference in risk for in-hospital mortality (adj. IRR 0.6 [95\% CI 0.3-1.2], $p=0.2$ ) and good functional outcome at discharge (GOS-E $\geq 5$ adj. IRR 1.0 [95\% CI 0.9-1.2], $p=0.8$ ) or 6 months following injury could be detected (GOS-E $\geq 5$ Adj. IRR: 1.1 [95\% CI 0.9-1.2], $p=0.3$ ).

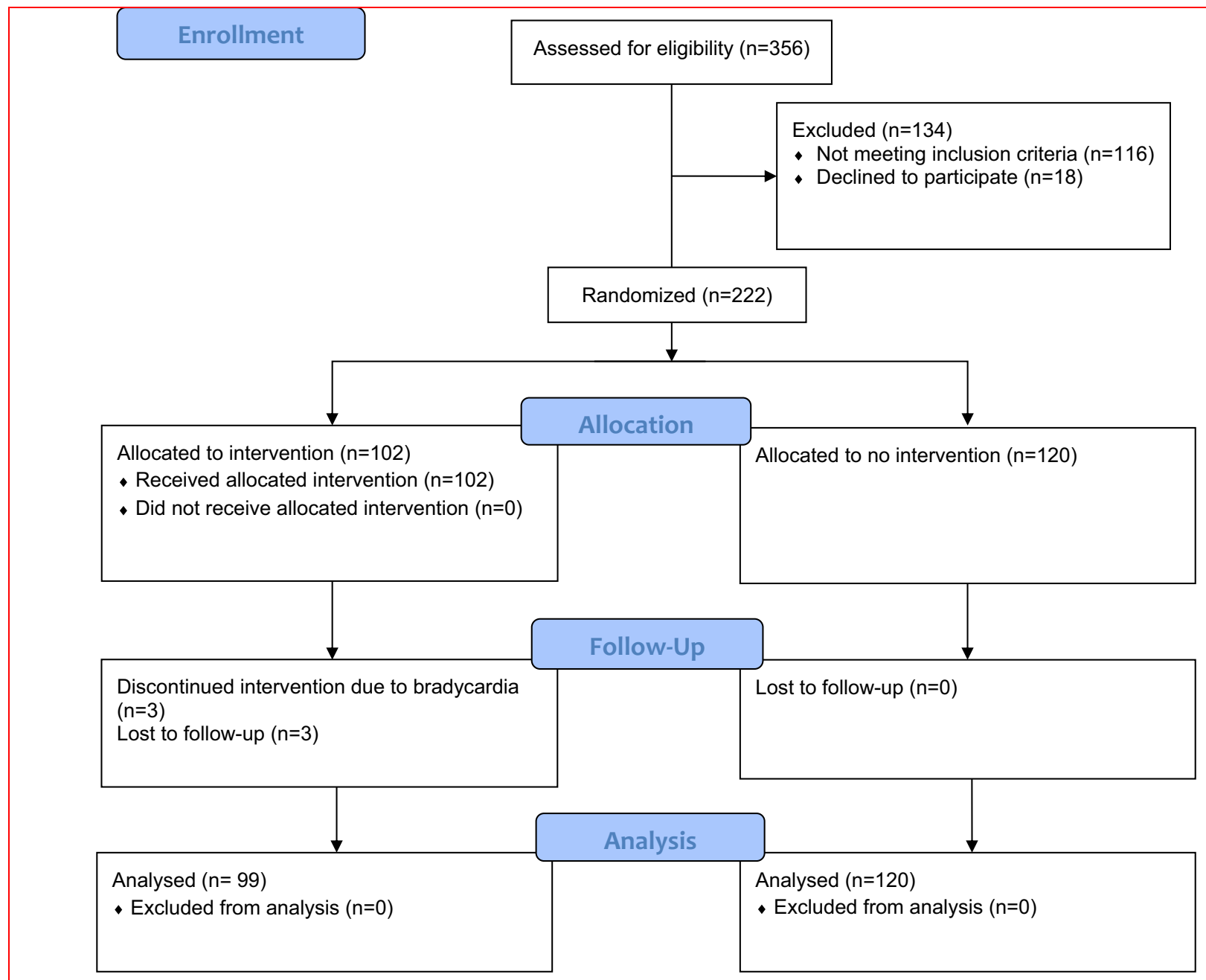

Fig. 1 Consort diagram of patient flow throughout the trial 
Table 1 Comparison of demographic and clinical characteristics between beta-blocker therapy positive and negative cohorts $(n=219)$

\begin{tabular}{|c|c|c|c|c|}
\hline & $\begin{array}{l}\text { Total } \\
n=219\end{array}$ & $\begin{array}{l}\mathrm{BB}(-) \\
n=120\end{array}$ & $\begin{array}{l}\mathrm{BB}(+) \\
n=99\end{array}$ & $p$ \\
\hline Male sex & $189(86.3)$ & $103(85.8)$ & $86(86.9)$ & 0.82 \\
\hline Age, mean $[\mathrm{SD}]$ & 38 [18] & $39[20]$ & 37 [17] & 0.34 \\
\hline Age $\geq 55$ years & $42(19.2)$ & $26(21.7)$ & $16(16.2)$ & 0.30 \\
\hline Hypertension & $22(10.0)$ & $13(10.8)$ & $9(9.1)$ & 0.67 \\
\hline Diabetes mellitus & $13(5.9)$ & $9(7.5)$ & $4(4.0)$ & 0.39 \\
\hline Cardiovascular disease & $16(7.3)$ & $9(7.5)$ & $7(7.1)$ & 0.90 \\
\hline SBP mmHg, mean [SD] & $130[18]$ & $129[19]$ & $131[17]$ & 0.57 \\
\hline $\mathrm{GCS} \leq 8$ & $82(37.4)$ & $49(40.8)$ & $33(33.3)$ & 0.25 \\
\hline Head AIS $\geq 4$ & $141(64.4)$ & $79(65.8)$ & $62(62.6)$ & 0.62 \\
\hline Thorax AIS $\geq 3$ & $55(25.1)$ & $30(25.0)$ & $25(25.3)$ & 0.97 \\
\hline Abdominal AIS $\geq 3$ & $9(4.1)$ & $5(4.2)$ & $4(4.0)$ & 1.0 \\
\hline Extremity AIS $\geq 3$ & $11(5.0)$ & $6(5.0)$ & $5(5.1)$ & 1.0 \\
\hline ISS median [IQR] & $22[16,29]$ & $25[16,29]$ & $22[17,27]$ & 0.20 \\
\hline ISS $\geq 16$ & $176(80.4)$ & $94(74.3)$ & $82(82.8)$ & 0.47 \\
\hline Epidural hemorrhage & $78(35.6)$ & $44(36.7)$ & $34(34.3)$ & 0.72 \\
\hline Subdural hemorrhage & $93(42.5)$ & $56(46.7)$ & $37(37.4)$ & 0.17 \\
\hline Subarachnoid hemorrhage & $80(36.5)$ & $45(37.5)$ & $35(35.4)$ & 0.74 \\
\hline Contusion & $128(58.4)$ & $74(61.7)$ & $54(54.5)$ & 0.29 \\
\hline Intraventricular hemorrhage & $12(5.5)$ & $8(6.7)$ & $4(4.0)$ & 0.55 \\
\hline Base of skull fracture & $121(55.3)$ & $70(58.3)$ & $51(51.5)$ & 0.31 \\
\hline Depressed skull fracture & $32(14.6)$ & $18(15.0)$ & $14(14.1)$ & 0.86 \\
\hline Pneumocephalus & $58(26.5)$ & $29(24.2)$ & $29(29.3)$ & 0.39 \\
\hline Craniectomy/craniotomy & $80(36.5)$ & $50(41.7)$ & $30(30.3)$ & 0.08 \\
\hline ICU LOS days, mean [SD] & $10[10]$ & $11[10]$ & $9[10]$ & 0.25 \\
\hline Median [interquartile range] & $7[3,15]$ & $7[3,16]$ & $6[3,12]$ & \\
\hline Hospital LOS days, mean [SD] & $15[13]$ & $14[12]$ & $16[14]$ & 0.40 \\
\hline Median [interquartile range] & $11[6,19]$ & $10[6,19]$ & $12[7,20]$ & \\
\hline Mortality & $28(12.8)$ & $20(16.7)$ & $8(8.1)$ & 0.058 \\
\hline GOS-E at discharge $\geq 5$ & $149(69.3)$ & $78(66.7)$ & $71(72.4)$ & 0.36 \\
\hline GOS-E at 6 months $\geq 5$ & $176(81.5)$ & $93(77.5)$ & $83(86.5)$ & 0.09 \\
\hline
\end{tabular}

Percentages are stated within brackets

$S B P$ admission systolic blood pressure, GCS Glasgow Coma Scale, AIS Abbreviated Injury Scale, ISS Injury Severity Score, IQR interquartile, $L O S$ length of stay, GOS-E Extended Glasgow Outcome Scale

After applying specified exclusion criteria to include only patients suffering from isolated severe TBI, a total of 154 patients were available for further analysis, $68(44 \%)$ patients in the $\mathrm{BB}^{+}$group and $86(56 \%)$ patients in the $\mathrm{BB}^{-}$group (see Fig. 2). Mean age was similar in the two groups with a mean age (standard deviation) of 35 (SD 16) years in $\mathrm{BB}^{+}$and 37 (SD 19) years in $\mathrm{BB}^{-}$patients $(p=0.6)$. The majority of patients were male with over $85 \%$ of the male sex in each subgroup. There were no significant differences in the prevalence of hypertension, diabetes mellitus or cardiovascular disease between the two groups (Table 2). Furthermore, no significant differences were seen between groups with regards to injury severity or type. Neurosurgical intervention (craniectomy/craniotomy) was required in $27.9 \%(n=19)$ in those receiving propranolol and in $38.4 \%(n=33)$ in those not treated with propranolol $(p=0.20)$ (Table 2$)$. While there was no significant difference in ICU or hospital length of stay, patients administered propranolol showed significantly reduced mortality compared to those unexposed (4.4\% vs. $18.6 \%, p=0.012$ ) (Table 2). Although there was no difference in good functional outcome at discharge $(p=0.08)$, the $\mathrm{BB}^{+}$group showed significantly better 
Fig. 2 Flow diagram of isolated severe TBI patient throughout the trial. *Isolated sTBI (severe traumatic brain injury) $=$ intracranial AIS $\geq 3$ with all extracranial injuries $\leq 2$

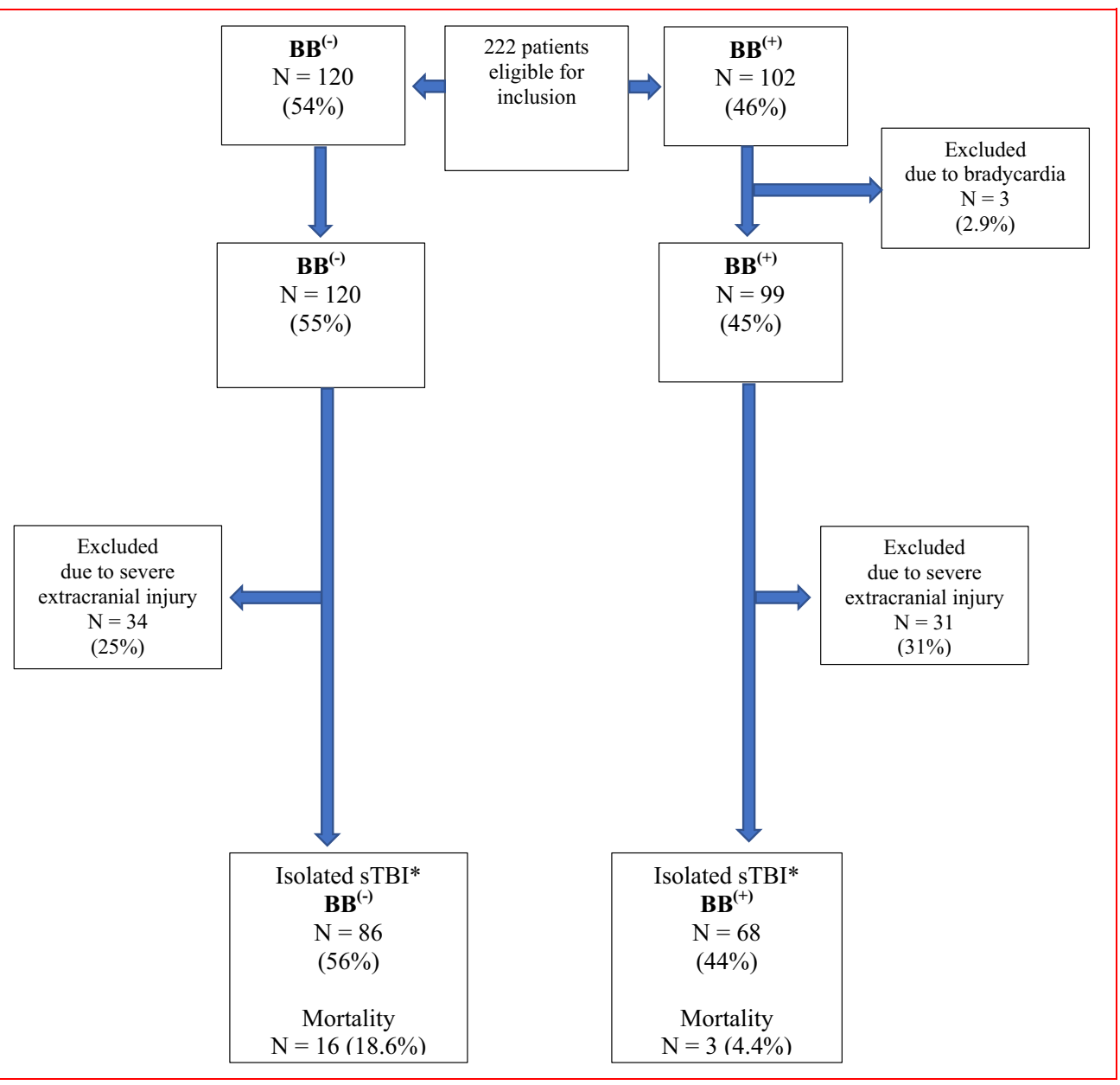

scores at 6-month follow-up (GOS-E $\geq 592.3 \%$ vs. $79.1 \%, p=0.04$ ) (Table 2).

Poisson regression analysis for in-hospital mortality outlined a strong protective link between propranolol and survival in patients suffering an isolated severe TBI. Patients not receiving propranolol had a threefold increase in mortality rate compared to those exposed (adj. IRR 3.1 [95\% CI 1.1-8.9], $p=0.037$ ) (Table 3). Additionally, factors demonstrating significant association with increased in-hospital mortality included age over 55 years, pre-injury hypertension, GCS score of eight or less at admission, ISS over 16, and the need for neurosurgical surgery (Table 3). The same regression model was carried out for functional outcome at discharge where no significant protective effect of beta-blockade could be detected (GOS-E $\geq 5$ adj. IRR 1.1 [95\% CI 0.9-1.3], $p=0.201$ ). At 6-month follow-up, however, propranolol-treated patients demonstrated a $20 \%$ relative improvement in good functional outcome (GOS$\mathrm{E} \geq 5$ adj. IRR 1.2 [95\% CI 1.0-1.3], $p=0.023$ ). A GCS of eight or lower on admission was predictive of poorer functional outcome at 6-month follow-up (GOS-E $\geq 5$ adj. IRR 0.80 [95\% CI 0.6-0.9], $p=0.005$ ) (Table 4).

\section{Discussion}

Whether the administration of regular beta-blockade should be part of the standardized treatment protocol in neurointensive care units for severe traumatic brain injuries is debated. While beta-blockade is not specifically recommended as part of the Brain Trauma Foundation guidelines, it is part of the so-called Lund concept guidelines developed by Lund University Hospital in Sweden [5]. The Lund concept recommends active lowering of systemic blood pressure through the administration of beta-blockade for brain volume control, optimization of brain perfusion, and oxygenation of the injured brain. Additionally, the Lund concept acknowledges the positive effect of the betablocker on the mitigation of the hyperadrenergic state. The guideline also recognizes the protective extracranial effects of beta-blockers by blocking the toxic effects mediated by TBI-induced catecholamine surges and vasogenic edema. Guidelines are often formed from multiple animal and retrospective clinical studies demonstrating improved outcomes following specific interventions, but no previous study has tested the Lund concept in a randomized 
Table 2 Comparison of demographic and clinical characteristics between beta-blocker therapy positive and negative cohorts with isolated severe TBI

\begin{tabular}{|c|c|c|c|c|}
\hline & $\begin{array}{l}\text { Total } \\
n=154\end{array}$ & $\begin{array}{l}\mathrm{BB}(-) \\
n=86\end{array}$ & $\begin{array}{l}\mathrm{BB}(+) \\
n=68\end{array}$ & $p$ \\
\hline Male sex & $132(85.7)$ & $74(86.0)$ & $58(85.3)$ & 0.90 \\
\hline Age, mean $[\mathrm{SD}]$ & $36[18]$ & 37 [19] & $35[16]$ & 0.60 \\
\hline Age $\geq 55$ years & $26(16.9)$ & $15(17.4)$ & $11(16.2)$ & 0.84 \\
\hline Hypertension & $16(10.4)$ & $9(10.5)$ & $7(10.3)$ & 0.97 \\
\hline Diabetes mellitus & $9(5.8)$ & $7(8.1)$ & $2(2.9)$ & 0.30 \\
\hline Cardiovascular disease & $9(5.8)$ & $6(7.0)$ & $3(4.4)$ & 0.73 \\
\hline SBP mmHg, mean [SD] & $130[19]$ & $130[20]$ & $130[17]$ & 0.90 \\
\hline $\mathrm{GCS} \leq 8$ & $46(29.9)$ & $29(33.7)$ & $17(25.0)$ & 0.24 \\
\hline Head AIS $\geq 4$ & $96(62.3)$ & $54(62.8)$ & $42(61.8)$ & 0.90 \\
\hline ISS median [IQR] & $20[13,25]$ & $20[13,26]$ & $20[15,24]$ & 0.20 \\
\hline ISS $\geq 16$ & $111(72.1)$ & $60(69.8)$ & $51(75.0)$ & 0.47 \\
\hline Epidural hemorrhage & $58(37.7)$ & $32(37.2)$ & $26(38.2)$ & 0.90 \\
\hline Subdural hemorrhage & $65(42.2)$ & $40(46.5)$ & $25(36.8)$ & 0.22 \\
\hline Subarachnoid hemorrhage & $47(30.5)$ & $29(33.7)$ & $18(26.5)$ & 0.33 \\
\hline Contusion & $82(53.2)$ & $50(58.1)$ & $32(47.1)$ & 0.17 \\
\hline Intraventricular hemorrhage & $9(5.8)$ & $7(8.1)$ & $2(2.9)$ & 0.30 \\
\hline Base of skull fracture & $87(56.5)$ & $50(58.1)$ & $37(54.4)$ & 0.64 \\
\hline Depressed skull fracture & $25(16.2)$ & $14(16.3)$ & $11(16.2)$ & 0.99 \\
\hline Pneumocephalus & $44(28.6)$ & $21(24.4)$ & $23(33.8)$ & 0.20 \\
\hline Craniectomy/craniotomy & $52(33.8)$ & $33(38.4)$ & $19(27.9)$ & 0.17 \\
\hline ICU LOS days, mean [SD] & 9 [9] & $10[9]$ & 7 [7] & 0.09 \\
\hline Median [interquartile range] & $5.5[3,12]$ & $6[3,15]$ & $5[3,10]$ & \\
\hline Hospital LOS days, mean [SD] & $14[13]$ & 14 [13] & $14[12]$ & 0.82 \\
\hline Median [interquartile range] & $10[6,18]$ & $9.5[6,19]$ & $11[6,18]$ & \\
\hline Mortality & $19(12.3)$ & $16(18.6)$ & $3(4.4)$ & 0.012 \\
\hline GOS-E at discharge $\geq 5$ & $109(70.8)$ & $56(65.1)$ & $53(77.9)$ & 0.08 \\
\hline GOS-E at 6 months $\geq 5$ & $128(84.8)$ & $68(79.1)$ & $60(92.3)$ & 0.04 \\
\hline
\end{tabular}

Percentages are stated within brackets

$S B P$ admission systolic blood pressure, GCS Glasgow Coma Scale, AIS Abbreviated Injury Scale, ISS Injury Severity Score, IQR interquartile, $L O S$ length of stay, GOS-E Extended Glasgow Outcome Scale

controlled trial or the alternative of no beta-blocker therapy in order to study the effects both short- and long-term overall outcomes. In spite of this, the Lund concept is used by several large university hospitals both in Sweden and in other countries. Conversely, the American Brain Trauma Foundation guidelines are used by other large university hospitals in the same countries.

Severe brain injury is strongly associated with catecholamine surge, often referred to as paroxysmal sympathetic storm. This hyperadrenergic activity is linked to increased risk of death through aggravating secondary brain injury and by inducing extracranial multiorgan dysfunction most notably cardiovascular, pulmonary and inflammatory $[13,21]$. Cerebral perfusion and subsequent oxygen delivery to cerebral tissue are impaired through catecholamine-induced cerebral vasoconstriction [11, 12]. Consequently, the use of beta-blockade to improve the cerebral environment in this context is drawn from physiological principles. The beneficial effects of beta-blockade on survival in TBI have been demonstrated in a multitude of retrospective cohort studies and were recently evaluated in a systematic review and meta-analysis by Chen et al. [22] demonstrating an odds ratio of $0.33(95 \%$ CI $0.27-0.40, p<0.001)$ for beta-blockade on in-hospital mortality. Similar survival advantages were detected by Alali et al. [23] after conducting a meta-analysis, and the authors conditionally recommend this treatment as part of Eastern Association for the Surgery of Trauma guideline 
Table 3 Incidence rate ratio for in-hospital mortality in patients with isolated severe TBI $(n=154)$

\begin{tabular}{lcc}
\hline & Adj. IRR $(95 \%$ CI $)$ & $p$ \\
\hline No beta-blocker therapy & $3.1(1.1-8.9)$ & 0.037 \\
Beta-blocker therapy & $0.32(0.1-0.9)$ & 0.037 \\
Male sex & $3.9(0.4-43.8)$ & 0.268 \\
Age $\geq 55$ years & $7.5(1.6-34.9)$ & 0.011 \\
Hypertension & $19.9(3.2-123.2)$ & 0.001 \\
Diabetes mellitus & $0.7(0.2-3.2)$ & 0.679 \\
GCS $\leq 8$ & $3.5(1.4-8.8)$ & 0.009 \\
Head AIS $\geq 4$ & $0.8(0.3-1.7)$ & $0.400 *$ \\
ISS $\geq 16$ & $13.9(4.2-45.6)$ & $<0.001$ \\
Epidural hemorrhage & $1.2(0.4-4.0)$ & 0.771 \\
Subdural hemorrhage & $0.8(0.3-1.9)$ & 0.577 \\
Subarachnoid hemorrhage & $1.8(0.6-5.3)$ & 0.289 \\
Contusion & $0.9(0.3-2.8)$ & 0.881 \\
Intraventricular hemorrhage & $0.8(0.1-8.1)$ & 0.887 \\
Base of skull fracture & $3.7(0.9-14.4)$ & 0.061 \\
Depressed skull fracture & $0.2(0.04-1.4)$ & 0.120 \\
Pneumocephalus & $2.2(0.9-5.2)$ & 0.079 \\
Craniectomy/craniotomy & $5.9(1.3-27.2)$ & 0.021 \\
\hline
\end{tabular}

*Not ajusted for ISS in the regression model

Table 4 Incidence rate ratio for good functional outcome (GOS-E $\geq 5$ ) at 6-month follow-up for patients with isolated severe TBI $(n=151)$

\begin{tabular}{lll}
\hline & Adj. IRR (95\% CI) & $p$ \\
\hline Beta-blocker therapy & $1.2(1.0-1.3)$ & 0.023 \\
Male sex & $0.9(0.8-1.0)$ & 0.269 \\
Age $\geq 55$ years & $0.6(0.4-0.9)$ & 0.026 \\
Hypertension & $0.8(0.5-1.2)$ & 0.281 \\
diabetes mellitus & $1.0(0.6-1.6)$ & 0.943 \\
Cardiovascular disease & $1.4(0.9-2.3)$ & 0.118 \\
GCS $\leq 8$ & $0.8(0.6-0.9)$ & 0.005 \\
Head AIS $\geq 4$ & $1.1(0.9-1.5)$ & $0.313 *$ \\
ISS $\geq 16$ & $0.8(0.6-1.1)$ & 0.119 \\
Epidural hemorrhage & $1.0(0.9-1.2)$ & 0.665 \\
Subdural hemorrhage & $1.1(0.9-1.2)$ & 0.329 \\
Subarachnoid hemorrhage & $0.9(0.7-1.0)$ & 0.096 \\
Contusion & $0.9(0.8-1.1)$ & 0.349 \\
Intraventricular hemorrhage & $1.2(0.9-1.6)$ & 0.200 \\
Base of skull fracture & $0.9(0.8-1.0)$ & 0.112 \\
Depressed skull fracture & $1.1(1.0-1.4)$ & 0.100 \\
Pneumocephalus & $0.9(0.7-1.1)$ & 0.215 \\
Craniectomy/craniotomy & $0.9(0.8-1.0)$ & 0.108 \\
\hline
\end{tabular}

*Not ajusted for ISS in the regression model for patients suffering a traumatic brain injury. The current study found that propranolol administered at $24 \mathrm{~h}$ after admission in patients with a severe isolated TBI was associated with a significant decrease in mortality (adj. IRR 0.32 [95\% CI 0.1-0.9], $p=0.037$ ).

The concept of neurocardiology is important in the context of severe TBI where the event of brain injury can lead to autonomic dysfunction and subsequent cardiac dysfunction, ischemia and arrhythmias [24-26]. Cardiac pathology is seen in up to $20 \%$ of patients with autopsies demonstrating similarities between cardiac injuries in TBI and in patients who die from a pheochromocytoma or a cocaine overdose [7]. Beneficial effects of beta-blockade on cardiac function following TBI has previously been demonstrated in a clinical trial and corroborated by a multitude of cohort studies [6, 27]. In a randomized clinical trial of 114 patients suffering acute head injury, atenolol was associated with significant reduction in the development of supraventricular tachycardia, ST segment and T wave changes and prevented cardiac necrosis seen at autopsy [27]. Of the observed deaths in the cohort of isolated severe TBIs in the current trial, 79\% $(n=15)$ were of cardiac nature. Only four deaths were of non-cardiac origin. Consequently, there is a physiological need for cardiac protection in TBI.

Although there are several studies indicating a survival benefit in TBI patients who have received beta-blockers during their hospitalization, there is a paucity in the evidence regarding any effect on long-term functional outcome. In a propensity-matched cohort of patients with severe TBI (intracranial AIS $\geq 3$ ), a strong association between pre-injury beta-blocker therapy with continuous in-hospital administration and better long-term functional outcome (GOS $\geq 4$ at 6 months post-discharge) was detected (57.9\% vs. $40.8 \%, p=0.03$ ) [28]. A possible underlying mechanism is an improved oxygenation and metabolism in the injured brain due to an improved cerebral circulation. In stroke patients, it has been shown that propranolol is associated with decreased oxygen and glucose consumption [15]. Similar results have been reproduced in vivo where propranolol increased the cerebral perfusion and decreased cerebral hypoxia [9]. These findings speak in favor of beta-blocker therapy which by altering the intracranial milieu may lead to better healing conditions of the injured brain tissue. In the current study, although no significant difference in good functional outcome (GOS-E $\geq 5$ ) could be detected at discharge ( $p=0.201)$, patients who received propranolol demonstrated a significant improvement in functional outcome at 6 months post-discharge (GOS-E $\geq 5$ adj. IRR 1.2, $p=0.023)$.

Concerns have, however, been raised regarding potential side effects of beta-blockade. The primary concern is the 
risk of hypotensive episodes. Hypotensive episodes in brain-injured patients have demonstrated significant association with both increased morbidity and mortality [29]. Additionally, the risk of bronchoconstriction due to beta-2 receptor blockade is another concern as this could lead to hypoxia and subsequent worsening of existing penumbra conditions due to the initial insult. In a prospective study by Murry et al. [18], the use of low-dose intravenous propranolol in patients suffering a mild-to-moderate TBI did not increase the number or severity of such events compared to controls. The safety of beta-blockade use after TBI in regard to adverse outcomes was also noted by Cruickshank and colleagues [27]. Interestingly, to date no increased risk for such adverse events has been published from centers that have introduced the Lund concept. In the current trial, no patients were excluded due to hypotension or hypoxia. Three patients were excluded on the basis of persistent bradycardia. None of them experienced any clinically significant adverse outcomes from this, and all three demonstrated good functional outcome at discharge.

Despite the prospective randomized design, the current trial is limited by a few study design factors. This includes the lack of blinding or placebo and the restriction to one trauma center only. Following patient exclusions to have a more homogenous study population including only severe isolated TBI, a total of 86 patients were analyzed in the beta-blocker-exposed group compared to only 68 patients in the beta-blocker-unexposed group. Finally, single doses of administered beta-blockade during the hospital stay were not controlled for. Consequently, it is possible that a minority of patients in the $\mathrm{BB}^{-}$group were given a single dose of a beta-blocker agent during the trial period.

\section{Conclusion}

The results of this trial show that the use of early oral propranolol in patients suffering isolated severe traumatic brain injury lead to improved survival and better functional outcome up to 6 months following injury. This provides support for the routine administration of beta-blocker therapy as part of a standardized neurointensive care protocol.

Acknowledgements Open access funding provided by Orebro University. We would like to thank our dedicated research nurses Ms. Fatemeh Abbasspour and Ms. Shadi Moghadam for their contribution with patient recruitment, randomization and daily data collection.

Author contributions HK, RA, SP, and SM were involved in study conception and design. HK, HAF, SP, AN helped in data acquisition. $\mathrm{HK}, \mathrm{RA}, \mathrm{GS}, \mathrm{BJ}, \mathrm{KH}, \mathrm{YC}, \mathrm{SM}$ contributed to the analysis and interpretation of data. HK, RA, SM were involved in the literature review. HK, RA, SP, GS, KH, BJ, SM helped in drafting the manuscript. All authors have critically reviewed and approved the final manuscript.

Funding The study received funding from research department of Shiraz University of Medical Sciences (Proposal ID\# 1396-01-3814792).

\section{Compliance with ethical standards}

Conflict of interest The authors have no conflicts of interest to disclose.

Open Access This article is licensed under a Creative Commons Attribution 4.0 International License, which permits use, sharing, adaptation, distribution and reproduction in any medium or format, as long as you give appropriate credit to the original author(s) and the source, provide a link to the Creative Commons licence, and indicate if changes were made. The images or other third party material in this article are included in the article's Creative Commons licence, unless indicated otherwise in a credit line to the material. If material is not included in the article's Creative Commons licence and your intended use is not permitted by statutory regulation or exceeds the permitted use, you will need to obtain permission directly from the copyright holder. To view a copy of this licence, visit http://creativecommons. org/licenses/by/4.0/.

\section{References}

1. Corrigan JD, Selassie AW, Orman JA (2010) The epidemiology of traumatic brain injury. J Head Trauma Rehabil 25(2):72-80

2. Chesnut RM, Marshall LF, Klauber MR et al (1993) The role of secondary brain injury in determining outcome from severe head injury. J Trauma 34:216-222

3. Manley G, Knudson MM, Morabito D et al (2001) Hypotension, hypoxia, and head injury: frequency, duration and consequences. Arch Surg 136(10):1118-1123

4. Carney $\mathrm{N}$ et al (2017) Guidelines for management of severe TBI, 4th edn. https://braintrauma.org/uploads/03/12/Guidelines_for_ Management_of_Severe_TBI_4th_Edition.pdf. Internet on May 112017

5. Koskinen LOD, Olivecrona M, Grände PO (2014) Severe traumatic brain injury management and clinical outcome using the Lund concept. Neuroscience 283:245-255

6. Alali AS, McCredie VA, Golan E et al (2014) Beta blockers for acute traumatic brain injury: a systematic review and metaanalysis. Neurocrit Care 20:514

7. Cotton BA, Snodgrass KB, Fleming SB et al (2007) Beta-blocker exposure is associated with improved survival after severe traumatic brain injury. J Trauma Injury Infect Crit Care 62(1):26-35

8. Ko A, Harada MY, Barmparas G et al (2016) Early propranolol after traumatic brain injury is associated with lower mortality. J Trauma Acute Care Surg 80(4):637-642

9. Dhillon NK, Inaba K, Salim A et al (2018) Beta blockers in critically ill patients with traumatic brain injury: results from a multicenter, prospective, observational American Association for the Surgery of Trauma study. J Trauma Acute Care Surg 84(2):234-244

10. Mohseni S, Talving P, Wallin G et al (2014) Pre-injury betablockade is protective in isolated severe traumatic brain injury. J Trauma 76(3):804-808 
11. Alexander RW, Davis JN, Lefkowitz RJ (1975) Direct identification and characterization of beta-adrenergic receptors in rate brain. Nature 258(5534):437-440

12. MacKenzie ET, McCulloch J, Harper AM (1976) Influence of endogenous norepinephrine on cerebral blood flow and metabolism. Am J Physiol 231(2):489-494

13. Heffernan DS, Inaba K, Arbabi S et al (2010) Sympathetic hyperactivity after traumatic brain injury and the role of $\beta$ blocker therapy. J Trauma 69(6):1602-1609

14. Hamill RW, Woolf PD, McDonald JV et al (1987) Catecholamines predict outcome in traumatic brain injury. Ann Neurol 21(5):438-443

15. Meyer JS, Okamoto Shimazu K et al (1974) Cerebral metabolic changes during treatment of subacute cerebral infarction by alpha and beta adrenergic blockade with phenoxybenzamine and propranolol. Stroke 5(2):180-195

16. Ley EJ, Sechnet J, Park R et al (2009) The in vivo effect of propranolol on cerebral perfusion and hypoxia after traumatic brain injury. J Trauma 66(1):154-161

17. Ley EJ, Clond MA, Bukur M et al (2012) Beta-adrenergic receptor inhibition affects cerebral glucose metabolism, motor performance, and inflammatory response after traumatic brain injury. J Trauma 73(1):33-40

18. Murry JS, Hoang DM, Barmparas G et al (2016) Prospective evaluation of early propranolol after traumatic brain injury. J Surg Res 200(1):221-226

19. Clifton GL, Ziegler MG, Grossman RG (1981) Circulating catecholamines and sympathetic activity after head injury. Neurosurgery $8(1): 10-14$
20. CONSORT Transparent Reporting of Trials. http://www.consortstatement.org/consort-statement/flow-diagram. Internet on Aug 212019

21. Kemp CD, Johnson JC, Riordan WP et al (2008) How we die: the impact of nonneurologic organ dysfunction in severe traumatic brain injury. Am Surg 74(9):866-872

22. Chen Z, Tang L, Xu X et al (2017) Therapeutic effect of betablocker in patients with traumatic brain injury: a systematic review and meta-analysis. J Crit Care 41:240-246

23. Alali AS, Mukherjee K, McCredie VA et al (2017) Beta-blockers and traumatic brain injury: a systematic review, meta-analysis, and Eastern Association for the Surgery of Trauma Guidelines. Ann Surg 266(6):952-961

24. Chen Z, Venkat P, Seyfried D et al (2017) Brain-heart interaction. Circ Res 121:451-468

25. Connor RC (1969) Myocardial damage secondary to brain lesions. Am Heart J 78(2):145-148

26. Piek J, Chesnut RM, Marshall LF et al (1992) Extracranial complications of severe head injury. J Neurosurg 77(6):901-907

27. Cruickshank Degaute JP, Kuurne T et al (1987) Reduction of stress/catecholamine-induced cardiac necrosis by beta1-selective blockade. Lancet 2(8559):585-589

28. Ahl R, Thelin EP, Sjolin G et al (2017) Beta-blocker after severe traumatic brain injury is associated with better long-term functional outcome: a matched case control study. Eur J Trauma Emerg Surg 43(6):783-789

29. Ghajar J (2000) Traumatic brain injury. Lancet 356:923-929

Publisher's Note Springer Nature remains neutral with regard to jurisdictional claims in published maps and institutional affiliations. 